\title{
Emergence of resistance after therapy with antibiotics used alone or combined in a murine model
}

\author{
J. C. Pechère, B. Marchon, M. Michéa-Hamzehpour and R. Auckenthaler \\ Departement de Microbiologie, Centre Médical Universitaire, 9 avenue de Champel, \\ 1211 Genève 4, Switzerland
}

\begin{abstract}
A murine model of peritonitis allowing detection and quantification of in-vivo acquired resistance during short term therapy has been used in order to evaluate the capacity of antimicrobial combinations to limit emergence of resistance, as compared to individual components of the regimens. Mice were challenged intraperitoneally with $10^{\mathrm{s}} \mathrm{cfu}$ of bacteria. Two hours later, a single antibiotic dose was injected subcutaneously: amikacin $(15 \mathrm{mg} / \mathrm{kg})$, ceftriaxone $(50 \mathrm{mg} / \mathrm{kg})$, pefloxacin $(25 \mathrm{mg} / \mathrm{kg})$, amikacin + ceftriaxone, amikacin + pefloxacin or ceftriaxone + pefloxacin. Escherichia coli and Staphylococcus aureus never became resistant. Single drug therapy yielded resistant mutants in Enterabacter cloacae, Serratia marcescens, Klebsiella pneumoniae and Pseudomonas aeruginosa as follows: $74 \%$ of ceftriaxone-treated animals, $57 \%$ of pefloxacin treated animals and $27 \%$ of amikacin treated animals. All the tested combinations reduced the frequency of in-vivo acquired resistance produced by single drugs, and no combination selected resistance when the separate agents of the combination did not. Combining antimicrobial agents limits the risk of emergence of resistance during antibiotic therapy.
\end{abstract}

\section{Introduction}

Antibiotic combinations are commonly used in clinical practice. Too often, the prescription of more than a single antimicrobial agent in a specific patient is not based upon scientifically established facts, but rather reflects the insecurity felt by clinicians confronted with life-threatening infections. However, in other cases, prognosis of severe infectious diseases is improved by combined antimicrobial therapy. To a large extent, the rationale of such therapy includes the wish to widen the antibacterial spectrum in mixed infections or in critically ill patients before identification of the invading pathogens or the desire to enhance antibacterial activity in some well-selected cases such as deep-seated pseudomonal infections or enterococcal endocarditis. Other reasons for using combinations have also been advocated, among them prevention of emergence of resistance. This represents a legitimate reason for using multiple drug therapy in tuberculosis, but in other bacterial infections it has been difficult so far to demonstrate similar beneficial effects (Klastersky, 1984).

However, acquired resistance to broad-spectrum antibiotics during therapy is a growing concern. The fact is well established with many of the newer $\beta$-lactam compounds (Sanders \& Sanders, 1985) and the threat is genuine with the recently developed fluoroquinolones which can easily produce resistant mutants in vitro (Chin \& Neu, 1983, 1984; Duckworth \& Williams, 1984; Sanders et al., 1984).

In this laboratory, a murine model of peritonitis allowing detection and quantification of in-vivo acquired resistance during short-term therapy has been used in order to 
evaluate the capacity of antimicrobial combinations to limit emergence of resistance, compared with components of the regimens used alone.

\section{Material and methods}

Antibiotics and strains. Ceftriaxone (Roche, Basel, Switzerland), pefloxacin (RhonePoulenc, Paris, France) and amikacin (Bristol-Myers, Cham, Switzerland) have been kindly provided in form of powders of known potency, which were dissolved in phosphate-buffered saline. The nine strains used in the study were clinical isolates, as listed in Table I. They were maintained in skimmed milk and stored at $-70^{\circ}$. Before study they were thawed and grown in L. broth.

Animal model. Swiss Webster female mice, weighing 20-30 g were used. Experimental peritonitis was established by intraperitoneal injection of $1 \mathrm{ml}$ of a mixture containing $10^{8} \mathrm{cfu}$ of a pure culture and $125 \mathrm{mg}$ of sterilized talcum in $\mathrm{NaCl}$ solution $0.9 \%$. Bacterial suspensions were prepared from 24-h cultures in L. broth. Two hours after challenge, the antimicrobial treatment was administered subcutaneously as a single dose in $0.2 \mathrm{ml}$, of $0.9 \% \mathrm{NaCl}$. The following dosage regimens were used : amikacin $15 \mathrm{mg} / \mathrm{kg}$, ceftriaxone $50 \mathrm{mg} / \mathrm{kg}$, pefloxacin $25 \mathrm{mg} / \mathrm{kg}$, amikacin $15 \mathrm{mg} / \mathrm{kg}+$ ceftriaxone $50 \mathrm{mg} / \mathrm{kg}$, amikacin $15 \mathrm{mg} / \mathrm{kg}$ + pefloxacin $25 \mathrm{mg} / \mathrm{kg}$, ceftriaxone $50 \mathrm{mg} / \mathrm{kg}$ + pefloxacin $25 \mathrm{mg} / \mathrm{kg}$. Twentyfour hours after challenge, animals were sacrificed by hyperanaesthesia. Peritoneal secretions were sampled through a $2-3 \mathrm{~mm}$ left iliac incision. Ten microlitres of peritoneal secretions were plated on $L$. agar for colony counting after appropriate dilutions, while the rest of the sample was placed into $L$. broth for further identification and bacterial population analysis.

Bacterial populations analysis was performed only on micro-organisms with the same identity (API system) as the strain originally inoculated. The peritoneal exudates grown in L. broth, after determination of the cfu, were diluted in order to provide $10^{8} \mathrm{cfu}(100 \mu \mathrm{l}$ plated on L. agar containing antibiotic gradient (Brysón \& Szybalski, 1952). Gradients were prepared in $9 \times 9 \mathrm{~cm}$ square Petri dishes, with the following concentrations $(\mathrm{mg} / \mathrm{l})$ : ceftriaxone $0-0.64,0-4,0-64,0-640$; amikacin $0-16,0-64$; pefloxacin $0-2$ or $0-32$. Antibiotic concentrations in the gradient vary from 0 to the chosen maxima as a linear function of the distance from the side of the dish where the gradients starts. After incubation for $24 \mathrm{~h}$ at $37^{\circ} \mathrm{C}$, bacterial growth was examined and, when possible, colonies counted. Typical appearances on these gradients include a confluent growth at lower antibiotic concentrations and non confluent colonies at higher concentrations. The quite sharp limit between these two zones has been called the boundary (Brysón \& Szybalsi, 1952). Preliminary comparative studies with our nine bacterial strains indicated that in the conditions specified above, the antibiotic concentration corresponding to the location of the boundary was always within two-fold the MIC determined by microdilution method using an inoculum of $10^{5} \mathrm{cfu} / \mathrm{ml}$ and Mueller Hinton broth. We have defined a significant shift towards resistance when at least one of the two following criteria was met (i) a greater than two-fold increase of the antibiotic concentration in the gradient corresponding to the boundary or (ii) greater than ten-fold increase of the number of colonies growing in the non confluent zone.

\section{Results}

One hundred and seventy nine animals were used in this study. At the time of sacrifice, peritoneal exudates contained $5 \times 10^{7}$ to $5 \times 10^{\circ} \mathrm{cfu} / \mathrm{ml}$ in the control animals, and always less in the treated animals (see the last column of Table I). In three instances, a 
Table I. Strains used in the study

\begin{tabular}{llccc}
\hline & & \multicolumn{3}{c}{ Original MICs (mg/l) of: } \\
\cline { 3 - 5 } Organisms & Clinical source & amikacin & ceftriaxone & pefloxacin \\
\hline E. coli 448 & urine & 1 & $<0.12$ & $<0.12$ \\
K. pneumoniae 24 & blood culture & 2 & 0.12 & 0.12 \\
Ent. cloacae 218 & CSF & 1 & 0.25 & 0.12 \\
Ent. cloacae 219 & CSF & 2 & 0.25 & 0.12 \\
Ent. cloacae 895 & mediastinum & 1 & 0.25 & 0.12 \\
Ent. cloacae 908* & blood culture & 1 & 0.25 & 0.25 \\
S. marsescens 240 & urine & 1 & 0.06 & 0.25 \\
P. aeruginosa 302 & urine & 8 & 4 & 2 \\
Staph. aureus 501 & blood culture & 0.12 & 0.5 & 0.25 \\
\hline
\end{tabular}

* Described by Then \& Anghern (1982).

contaminant was found in the peritoneum, while in three animals, culture of the peritoneal exudates yielded no growth. These six animals were excluded from the study. The treatment resulted in a reduction of the bacterial populations by one to seven logs (Table II). The cfu decreases were similar for the three antibiotics used as single agents against all enterobacters, Escherichia 448 , Serratia marcescens 240 and Staphylococcus aureus 501 . Pefloxacin was found to be less active than amikacin or ceftriaxone against Klebsiella pneumoniae 24, but more active against Pseudomonas aeruginosa 302 . Combinations produced a more potent effect than monotherapy by $10-10^{6}$ times (Table II). Exceptions to this general pattern were observed with $K$. pneumoniae 24 , where monotherapy by amikacin and ceftriaxone was not improved by combinations. This may be due to an especially potent effect obtained with the antibiotics alone $\left(10^{5}-10^{7}\right.$ less colonies than in controls), leaving no possibility for improvement with combinations.

Results of Szybalski's gradient were analysed to determine the frequency of resistance acquired after the different therapeutic regimens. Single-shot treatments allowed easy selection of resistant mutants in Ent. cloacae, $P$. aeruginosa, $S$. marcescens and $K$. pneumoniae, but never in mice challenged with E. coli or Staph. aureus (Table II). Significant shift was most commonly observed after treatment with ceftriaxone, where five out of the nine strains developed resistance, the four enterobacters and $P$. aeruginosa 302. In total, ceftriaxone yielded selection of resistant mutants in 42 of the 57 ceftriaxone-treated mice $(74 \%)$. When a significant shift occurred after ceftriaxone therapy the MIC (according to the location of the boundary) increased 128- to 1024-fold with the enterobacters, and only four-fold with P. aeruginosa 302. In the case of Ent. cloacae 219, ceftriaxone therapy selected a ceftriaxone-resistant mutant which also expressed a four-fold reduction in susceptibility to amikacin. In animals treated with pefloxacin, emergence of mutants with a four- to 16-fold reduction in susceptibility occurred in 4 strains, $K$. pneumoniae 24, Ent. cloacae 128, S. marcescens 240 and $P$. aeruginosa 302 . In this last instance, the mutant resistant to pefloxacin was also resistant to ceftriaxone. Overall, pefloxacin was responsible for 13 significant shifts among the 23 pefloxacin-treated mice (57\%). Four strains, Ent cloacae 218 and 895, S. marcescens 240 and $P$. aeruginosa 302 , engendered significant shifts on amikacin gradients after therapy. Significant shifts were not associated with MIC increase but were characterized by a 15-fold increase of the number of colonies growing in the non confluent zone on the Szybalski's gradient. In the $\mathbf{2 2}$ mice treated with amikacin, this aminoglycoside produced six significant shifts $(27 \%)$. 


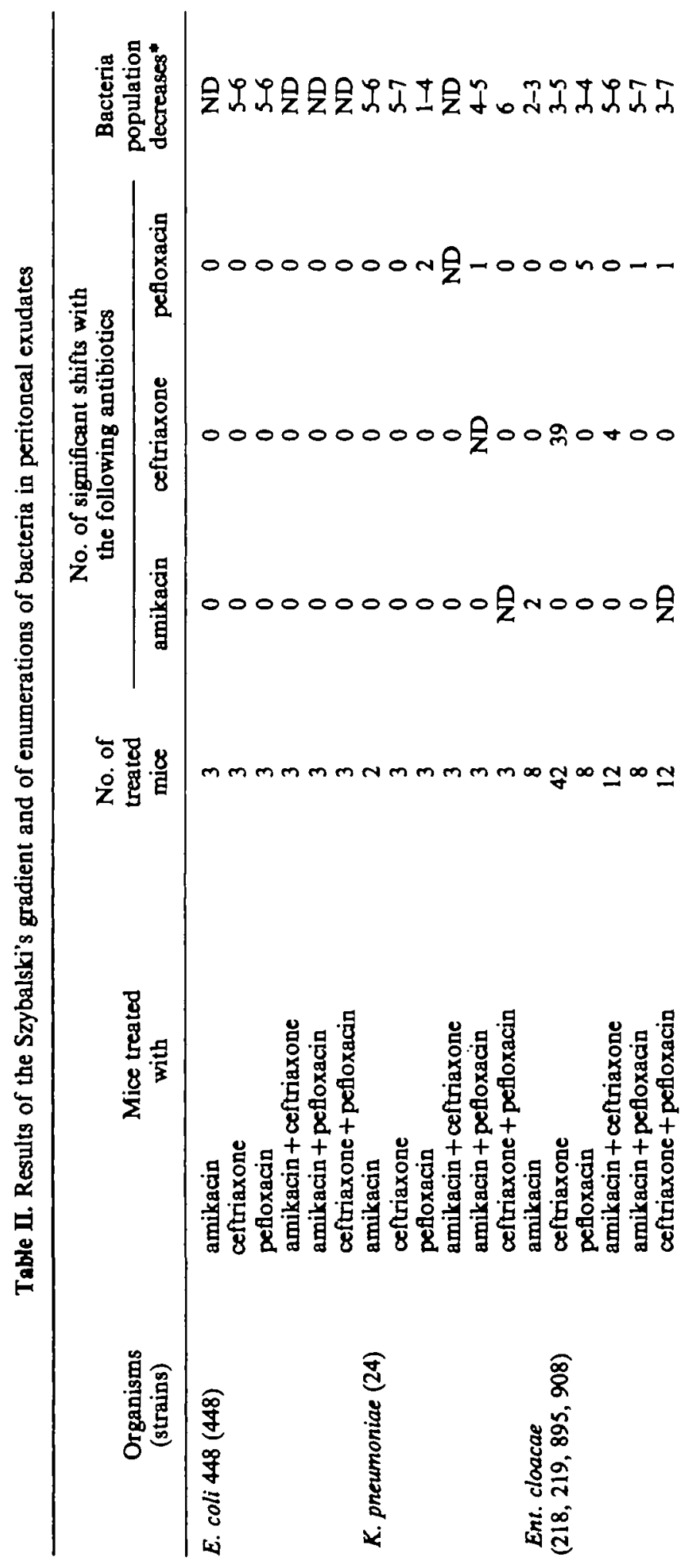




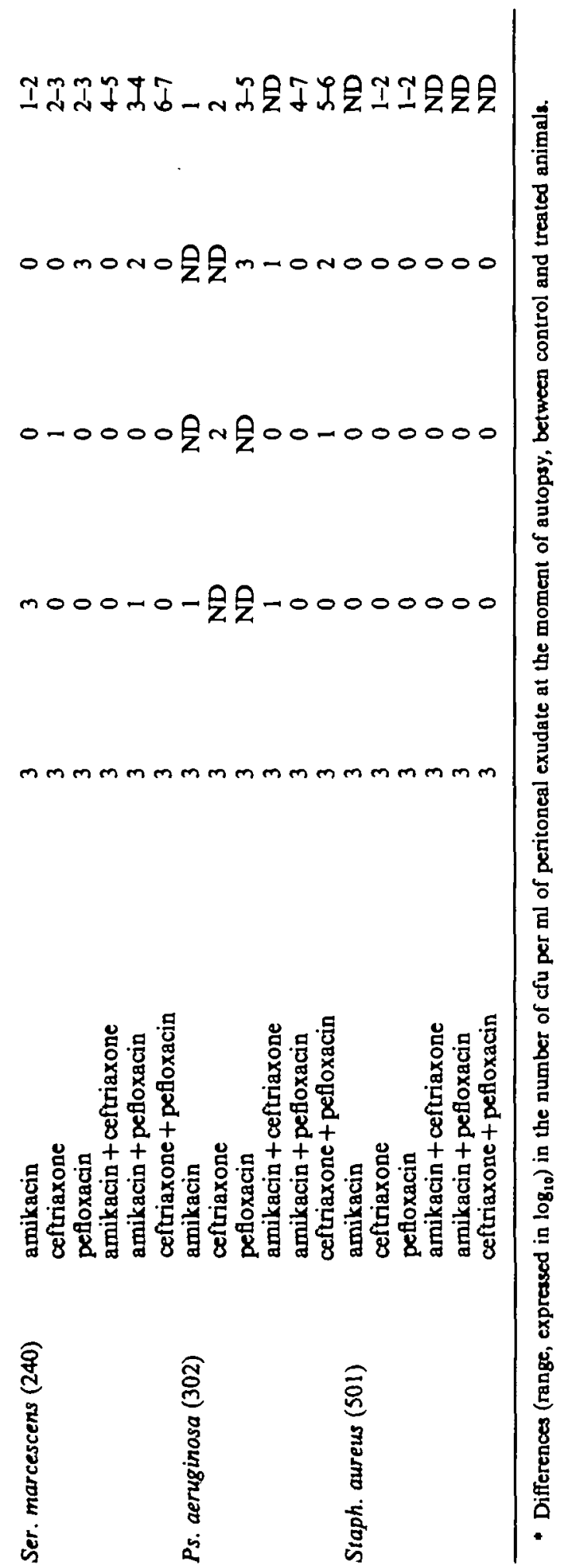


Table III. MIC increases after passage in treated animals

\begin{tabular}{|c|c|c|c|c|}
\hline \multirow[b]{2}{*}{$\begin{array}{l}\text { Strains inoculated } \\
\text { into the animal }\end{array}$} & \multirow[b]{2}{*}{$\begin{array}{l}\text { Treatment given } \\
\text { to the animal }\end{array}$} & \multicolumn{3}{|c|}{$\begin{array}{l}\text { Fold increase of MIC in treated } \\
\text { animals as compared to controls }\end{array}$} \\
\hline & & amikacin & $\begin{array}{l}\text { with } \\
\text { ceftriaxone }\end{array}$ & pefloxacin \\
\hline \multirow[t]{3}{*}{ K. pneumoniae 24} & amikacin & 1 & 1 & 1 \\
\hline & ceftriax one & 2 & 1 & 1 \\
\hline & pefloxacin & 1 & $i$ & 4 \\
\hline \multirow[t]{3}{*}{ Ent. cloacae 218} & amikacin & 1 & 1 & 1 \\
\hline & ceftriax one & 2 & 128 & 1 \\
\hline & pefloxacin & 1 & 1 & 4 \\
\hline \multirow{3}{*}{ Ent. cloacae 219} & amikacin & 2 & 1 & 1 \\
\hline & ceftriax one & 2 & 1024 & 1 \\
\hline & pefloxacin & 1 & 1 & $i$ \\
\hline \multirow[t]{3}{*}{ Ent. cloacae 895} & amikacin & $i$ & $i$ & 1 \\
\hline & ceftriaxone & 1 & 1024 & 1 \\
\hline & pefloxacin & 1 & 1 & 1 \\
\hline \multirow[t]{3}{*}{ Ent. cloacae 908} & amikacin & 1 & 1 & 1 \\
\hline & ceftriaxone & 1 & 512 & 1 \\
\hline & pefloxacin & 1 & 1 & 1 \\
\hline \multirow[t]{3}{*}{ S. marcescens 240} & amikacin & 1 & 1 & 1 \\
\hline & ceftriaxone & 1 & 1 & 1 \\
\hline & pefloxacin & 1 & 1 & 16 \\
\hline \multirow[t]{3}{*}{$P$. aeruginosa 302} & amikacin & 1 & 1 & 1 \\
\hline & ceftriaxone & 1 & 4 & 1 \\
\hline & pefloxacin & $i$ & 16 & 16 \\
\hline
\end{tabular}

No MIC increases were observed with E. coli 448 and Stoph. aureus S01.

In all cases, combinations selected less resistant mutants than the components of the regimens used alone. In 21 animals, amikacin plus ceftriaxone produced only one shift on the amikacin gradient $(5 \%)$ and four shifts on the ceftriaxone gradient $(19 \%)$. In the 17 mice treated with amikacin and pefloxacin, one $(6 \%)$ and four $(23 \%)$ shifts respectively were found on the gradients prepared with amikacin and pefloxacin. Finally, in 21 animals given the combination ceftriaxone-pefloxacin, one shift appeared on the ceftriaxone gradient $(5 \%)$ and three others on the pefloxacin gradient $(14 \%)$. However, according to the magnitude of the 'boundary' shift on the gradients, the antibiotic concentrations required for inhibiting the resistant mutants selected by combinations were similar to those determined in the case of selection by single agents. It never happened that a combination yielded a significant shift when the agents of the combination did not. In that sense, no antagonism occurred.

\section{Discussion}

Development of resistance during single-drug therapy with ceftriaxone, amikacin and pefloxacin in the experimental model used in this study is quite similar to that reported in previous in-vitro studies (Sanders et al., 1984). To some extent, this also corresponds to clinical experience. In the case of amikacin, emergence of resistance during monotherapy is not clearly documented, probably because this agent is usually used in combination. In contrast, during recent years, emphasis has been laid on the ability of 
certain Gram-negative bacilli to develop resistance rapidly to many of the newer $\beta$-lactam antibiotics. The phenomenon was first stressed for cefamandole (Sanders et al., 1982), but today, the list of compounds for which similar disadvantage has been reported include nearly all the recently-developed 'enzyme stable' $\beta$-lactams. The bacteria that possess the ability to become rapidly resistant to these antibiotics during therapy include such genera as Enterobacter, Pseudomonas, Serratia, Citrobacter and Acinetobacter.

With the newer quinolones, similar problems seem to exist, even though, due to the lack of sufficient clinical experience, resistance developing during therapy with these drugs is not so well documented as in the case of $\beta$-lactam antibiotics, but it was well known with older structurally-related drugs. Many early studies with nalidixic acid and later with oxolinic acid describe treatment failures associated with selection of resistant mutants. Accumulated results from 5 trials of nalidixic acid (Slack, 1984) showed 132 failures out of 348 completed courses; of this $39 \%$ failure rate, $12 \%$ of patients had a resistant organism of the same species as the initial sensitive one. Also it has been said that $7 \%$ of patients treated with $4 \mathrm{~g}$ /day of nalidixic acid will develop resistant organisms in the urinary tract (Stamey, 1980). However in-vitro selection of resistant mutants during therapy appears less frequent with the newer fluoroquinolones than with the parent compounds (Sanders et al., 1984). In addition, due to the improved antibacterial activity of drugs such as pefloxacin and ciprofloxacin, the resistant variants, although less sensitive than the parent strains, might still be regarded as sensitive to these drugs (King, Shannon \& Phillips, 1984). Unfortunately, preliminary clinical experience indicates that selection of resistant clones during therapy with fluoroquinolones exists. In 28 patients treated with intravenous ciprofloxacin, the susceptibility of the pathogen decreased rapidly by several hundred-fold in three instances (Helfer, R., Auckenthaler \& Waldvogel, personal communication). Very interestingly, the bacteria that developed such resistance belonged to genera already involved in the resistance to newer $\beta$-lactams: $P$. aeruginosa and $S$. marcescens. Clinical experience also teaches that monotherapy can select mutants showing resistance not only to the drug administered to the patient, but also to other antibiotics belonging to unrelated classes. In their clinical study on moxalactam for instance, Preheim et al. (1982) reported the emergence of two strains of $P$. aeruginosa with reduced susceptibility to both $\beta$-lactams and aminoglycosides.

In contrast to the overwhelming data concerning monotherapy, emergence of resistance with combinations is much more rarely mentioned in the literature. Examples exist however, as in the bacteraemia caused by a strain of $S$. marcescens which gave rise to mutants resistant to the two antibiotics given to patient, cefotaxime and amikacin reported by Goldstein $e t$ al. (1983). The relative scarcity of reports dealing with similar experiences contrasts with the great number of infected patients receiving two antibiotics, but fits with the results of this study, indicating that combinations notably reduce the risk of resistance emerging during therapy.

The lesser risk of resistance observed with combinations is probably associated with relative frequencies of resistant variants in bacterial populations before exposure to the antibiotics, as seen on the Szybalski's gradient. In this study, analysis of the gradient plated with wild-type bacteria allowed to some extent a forecast of further occurrence of resistance in the treated mice. More colonies growing on areas corresponding to higher antibiotic concentrations were more likely to produce a significant shift after passage in mice treated with the same drug. The mutational frequencies for resistance to a single antibiotic ranged from $10^{-}$to $10^{-0}$ in the wild bacteria but the probability of selection of mutants able to resist simultaneously two structurally unrelated antibiotics was certainly lower. Actually such cross-resistance was found only once in this study 
contrasting with the ease of obtaining resistance to only one agent. The mechanisms producing cross resistances between quinolones, aminoglycosides and $\beta$-lactams remain hypothetical, but often multiple drug resistance involves a permeability change, as shown previously in $S$. marcescens (Gutmann \& Chabbert, 1984), P. aeruginosa (Rella \& Hass, 1982), K. pneumoniae (Sanders et al., 1984, Gutmann et al., 1985), and Ent. cloacae (Gutmann et al., 1985).

\section{Acknowledgement}

This study was supported by the "Fonds National Suisse de la Recherche" (grant no $3 \cdot 544-0 \cdot 83$ ).

\section{References}

Brysón, L. \& Szybalski, W. (1952). Microbial selection. Sciences 116, 45-51.

Chin, N. X. \& Neu, H. C. (1984). Ciprofloxacin, a quinolone carboxylic acid compound active against aerobic and anaerobic bacteria. Antimicrobial Agents and Chemotherapy 25, 319-26.

Duckworth G. J. \& Williams J. D. (1984). Frequency of appearance of resistant variants to norfloxacin and nalidixic acid. Journal of Antimicrobial Chemotherapy 13, Suppl. B, 33-8.

Goldstein, F. W., Gutmann, L., Williamson, R., Collatz, E. \& Acar, J. F. (1983). In vivo and in vitro emergence of simultaneous resistance to both betalactam and aminoglycoside antibiotics in a Serratia marcescens. Annales de Microbiologie 134A, 329-37.

Gutmann, L. \& Chabbert, Y. A. (1984). Different mechanisms of resistance to latamoxef (moxalactam) in Serratia marcescens strains. Journal of Antimicrobial Chemotherapy 13, $15-22$.

Gutmann, L., Williamson, R., Moreau, N., Kitzis, M. D., Collatz, E., Acar, J. F. \& Goldstein, F. W. (1985). Cross resistance to nalidixic acid, trimotheprim, and chloramphenicol associated with alterations in outer membrane proteins of Klebsiella, Enterobacter and Serratia. Journal of Infectious Diseases 151, 501-7.

King, A., Shannon, K. \& Phillips, I. (1984). The in vitro activity of ciprofloxacin compared with that of norfloxacin and nalidixic acid. Journal of Antimicrobial Chemotherapy 13, 325-31.

Klastersky, J. (1984). Antibiotic synergy and antagonism. In Antimicrobial Therapy, pp. 37-53. Raven Press, New York.

Preheim, L. C., Penn, R. G., Sanders, C. C., Goering, R. V. \& Giger, D. K. (1982). Emergence of resistance to betalactam and aminoglycoside antibiotics during moxalactam therapy of Pseudomonas aeruginosa infections. Antimicrobial Agents and Chemotherapy 22, 1037-41.

Rella, M. \& Haas, D. (1982). Resistance of Pseudomonas aeruginosa PAO to nalidixic acid and low levels of beta-lactam antibiotics: mapping of chromosomal genes. Antimicrobial Agents and Chemotherapy 22, 242-9.

Sanders, C. C., Moellering, R. C. Jr., Martin, R. R., Perkins, R. L., Strike, D. G., Gootz, T. D. \& Sanders, W. E. Jr. (1982). Resistance to cefamandole: a collaborative study of emerging clinical problems. Journal of Infectious Diseases 145, 118-25

Sanders, C. C. \& Sanders, W. E. Jr. (1985). Microbial resistance to newer generation betalactam antibiotics: clinical and laboratory implications. Journal of Infectious Diseases 151, 399-406.

Sanders, C. C., Sanders, W. E., Goering, R. V. \& Werner, V. (1984). Selection of multiple antibiotic resistance by quinolones betalactams and aminoglycosides with special reference to cross resistance between unrelated drug classes. Antimicrobial Agents and Chemotherapy 26, 797-801.

Slack, R. (1984). Review of bacterial resistance - a challenge to the treatment of urinary infection. Journal of Antimicrobial Chemotherapy 13, Suppl. B, 1-7.

Stamey, T. A. (1980). The pathogenesis and treatment of urinary tract infections. Williams \& Wilkins, Baltimore.

Then, R. L. \& Anghern, P. (1982). Trapping of non hydrolyzable œphalosporins by cephalosporinases in Enterobacter cloacae and Pseudomonas aeruginosa as a possible resistance mechanism. Antimicrobial Agents and Chemotherapy 21, 711-7. 